\title{
Contribution à l'étude des paramètres dendrométriques et à la cartographie des peuplements relictuels d'une espèce septentrionale rare au Maroc : Alnus glutinosa (L.) Gaertn.
}

Hassan EnNouni ${ }^{1}$

Abdelouahab SAHLI ${ }^{1}$

Mohammed ATER ${ }^{1}$

${ }^{1}$ Université Abdelmalek-Essaâdi Faculté des sciences Département de biologie

Laboratoire de botanique appliquée

Équipe Bio-Agrodiversité

BP 2062

93030 Tétouan

Maroc

Auteur correspondant /

Corresponding author:

Mohammed ATER - m.ater@uae.ac.ma

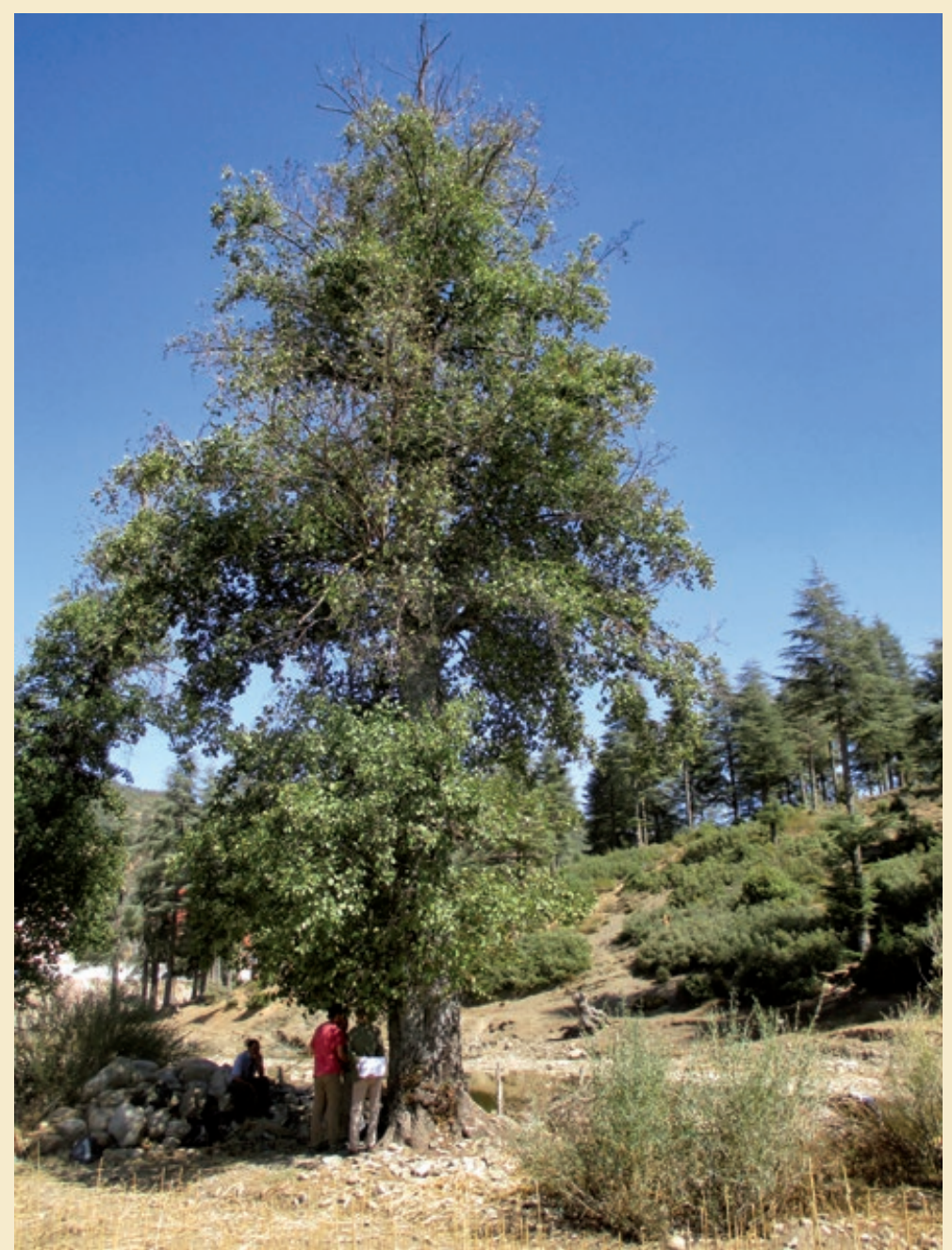

Photo 1.

Beau spécimen d'Alnus glutinosa dans un cours d'eau d'altitude, Oued Ketama dans la cédraie d'lkaouen non loin de Tlata Ketama (Rif central).

Beautiful specimen of Alnus glutinosa in a high altitude stream, Oued Ketama in the cedar forest of Ikaouen not far from Tlata Ketama (central Rif).

Photo M. Ater.

Doi : 10.19182/bft2021.349.a36777 - Droit d'auteur (c) 2021, Bois et Forêts des Tropiques - (c) Cirad - Date de soumission : 3 décembre 2020 ; date d'acceptation : 30 avril 2021 ; date de publication : $1^{\text {er }}$ septembre 2021.

\section{Ccirad (a)}

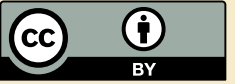

Citer l'article / To cite the article

Ennouni H., Sahli A., Ater M., 2021. Contribution à l'étude des paramètres dendrométriques et à la cartographie des peuplements relictuels d'une espèce septentrionale rare au Maroc : Alnus glutinosa (L.) Gaertn. Bois et Forêts des Tropiques, 349 : 21-35. Doi : https://doi.org/10.19182/ bft2021.349.a36777 


\section{RÉSUMÉ}

\begin{abstract}
Contribution à l'étude des paramètres dendrométriques et à la cartographie des peuplements relictuels d'une espèce septentrionale rare au Maroc : Alnus glutinosa (L.) Gaertn.
\end{abstract}

Alnus glutinosa (L.) Gaertn. (Betulaceae) est une espèce clé dans certaines formations alluviales et riveraines du continent européen. Au Maroc, elle se trouve en limite méridionale de son aire de distribution où elle est représentée par des formations relictes dans les zones refuges où elle a migré pendant les phases glaciaires du Pléistocène. Malgré sa grande valeur patrimoniale, il y a très peu de données sur son aire de répartition et l'état des peuplements. Ce travail a permis de localiser et cartographier les principaux peuplements dans la zone d'occurrence de cette espèce dans le Rif au nord du Maroc. La typologie des peuplements a été réalisée dans douze sites de référence en déterminant leurs principales caractéristiques telles que la nature du régime forestier, la composition, la taille, le recouvrement et la densité. Les caractéristiques dendrométriques ont permis de renseigner la structure et la dynamique des peuplements. Les résultats obtenus constituent un apport important de données quantitatives géoréférencées sur la répartition actuelle et la dynamique des peuplements d'A. glutinosa au Maroc. Étant donné l'état de conservation des peuplements, la dégradation de l'habitat et la faible régénération, cette espèce peut être considérée comme menacée et doit bénéficier de mesures urgentes de protection.

Mots-clés : aulne glutineux, Alnus glutinosa, limite d'aire, ripisylve, cartographie, dendrométrie, conservation, Rif centro-occidental, Maroc.

\section{ABSTRACT}

Contribution to studies on dendrometric parameters and mapping of relict stands of Alnus glutinosa (L.) Gaertn., a northern species rarely found in Morocco

The common alder, Alnus glutinosa (L.) Gaertn. (Betulaceae), is a key species in certain alluvial and riparian formations in continental Europe. The alders found in Morocco are at the southernmost boundary of their range and represented there by relict formations in refuge zones to which the species had migrated during the glacial phases of the Pleistocene. Despite its heritage value, there are very few data on its range of distribution or the condition of its populations. This study aimed to locate and map the main forest stands where this species occurs in the Rif region of northern Morocco. A typology of the stands was produced from surveys of twelve reference sites by determining their main characteristics, including stand composition, size, coverage, density and the nature of the forest regime. Stand structure and dynamics were determined from these dendrometric characteristics. The results contribute important quantitative and georeferenced data on the current distribution and dynamics of $A$. glutinosa populations in Morocco. Given their poor state of conservation, their degraded habitats and low regeneration rate, this species should be considered as endangered and urgently in need of protective measures.

Keywords: common alder, Alnus glutinosa, range boundary, riparian forest, mapping, dendrometrics, conservation, Central-Western Rif, Morocco.

\section{RESUMEN}

Contribución al estudio de los parámetros dendrométricos y a la cartografía de los relictos de una especie rara del norte de Marruecos: Alnus glutinosa (L.) Gaertn.

Alnus glutinosa (L.) Gaertn. (Betulaceae) es una especie clave en algunas formaciones aluviales y ribereñas del continente europeo. En Marruecos se encuentra en el límite sur de su área de distribución, donde está representada por formaciones relictas en las zonas de refugio a las que migró durante las fases glaciares del Pleistoceno. A pesar de su alto valor patrimonial, existen muy pocos datos sobre su área de distribución y el estado de conservación de la masa forestal. Este trabajo ha permitido localizar y cartografiar las principales masas forestales en la zona donde se encuentra esta especie en el Rif, en el norte de Marruecos. La tipología de las poblaciones se llevó a cabo en doce lugares de referencia determinando sus principales características, como la naturaleza del régimen forestal, la composición, el tamaño, la cobertura y la densidad. Las características dendrométricas proporcionaron información sobre la estructura y la dinámica de las masas. Los resultados obtenidos constituyen una importante aportación de datos cuantitativos georreferenciados sobre la distribución y la dinámica actuales de las poblaciones de $A$. glutinosa en Marruecos. Dado el estado de conservación de las masas, la degradación del hábitat y la escasa regeneración, esta especie puede considerarse amenazada y debe contar con medidas de protección urgentes.

Palabras clave: aliso, Alnus glutinosa, límite del área de distribución, bosque de ribera, cartografía, dendrometría, conservación, Rif centro-occidental, Marruecos. 


\section{Introduction}

Alnus glutinosa (L.) Gaertn. (Betulaceae) est un arbre monoïque caducifolié. II s'agit d'une espèce de souche eurasiatique dont l'aire s'étend de la Sibérie occidentale aux montagnes de la Turquie et de l'Afrique du Nord (McVean, 1953). Elle est répartie dans des formations alluviales et riveraines du continent européen, dans les basses terres et les altitudes moyennes. En Afrique du Nord, sur la rive méridionale de la région méditerranéenne, elle est en limite de son aire de distribution. Au Maroc, cette espèce se trouve dans le Rif centro-occidental, région reconnue parmi les points chauds de la biodiversité méditerranéenne (Médail et Quézel, 1999) et considérée comme un carrefour biogéographique où se rencontrent différentes influences floristiques, méditerranéennes, eurasiennes et subtropicales (Arènes, 1951 ; Quézel, 2000). La présence de l'aulne dans cette région s'explique par les migrations vers les zones refuges au sud de l'aire de l'espèce pendant les phases glaciaires du Pléistocène (Lepais et al., 2013 ; Douda et al., 2014).

Ainsi, les populations marocaines se trouvent isolées en marge de l'aire de l'espèce avec une histoire démographique et évolutive complexe. La différenciation génétique par l'isolement se traduit par la polyploïdisation, qu'elles partagent avec d'autres populations en limite d'aire, en péninsule ibérique et dans les Alpes dinariques (Lepais etal., 2013 ; Havrdová et al., 2015). Des travaux récents (Vit et al., 2017) ont même proposé de créer un nouveau taxon de rang spécifique, Alnus lusitanica, pour les populations ibériques et marocaines. D’une manière générale, la comparaison des populations diploïdes $d$ 'A. glutinosa avec les populations tétraploïdes montre que ces dernières présentent une plus grande richesse allélique et une diversité génétique plus élevée, marquant une nette distinction génétique au sein de l'espèce (Havrdová et al., 2015 ; Mandák et al., 2016). La résilience de ces populations face aux changements climatiques du passé leur confère une importance particulière pour les approches prédictives dans le contexte des changements climatiques déjà en cours. Ces populations doivent avoir un statut prioritaire pour la conservation eu égard à leur intérêt biogéographique, leur diversité et leur singularité génétique, ainsi que leur potentiel adaptatif face aux changements climatiques.

Au Maroc, bien que $A$. glutinosa soit considéré comme une espèce rare (Fennane et Ibn Tattou, 1998) et quasi menacée nécessitant des mesures de protection (Fennane, 2017), cet arbre ne bénéficie d'aucune mesure de protection et les données sur la répartition et la dynamique de ses populations sont inexistantes. Pourtant, cette espèce occupe des habitats vulnérables, principalement dans des formations rivulaires le long des cours d'eau. Dans son contexte local, elle est surexploitée même si elle n'offre pas de véritables opportunités de valorisation commerciale (Benamar, 2005). En effet, son usage porte sur la production d'unités fourragères en période de disette par la pratique de l'émondage, de la récolte de bois de chauffage et la fabrication de certains articles domestiques (ustensiles de cuisine, planches pour écriture des écoles coraniques, mancherons et clous de charrue...).
Ce travail représente une contribution à la connaissance de la répartition et de la dynamique des peuplements de l'aulne glutineux, $A$. glutinosa, au Maroc et constitue un apport important à d'éventuelles approches de conservation. En effet, en plus de la localisation et la cartographie des aulnaies, les travaux réalisés dans cette étude permettent de connaître la structure et la dynamique des peuplements à la faveur de l'analyse des paramètres dendrométriques.

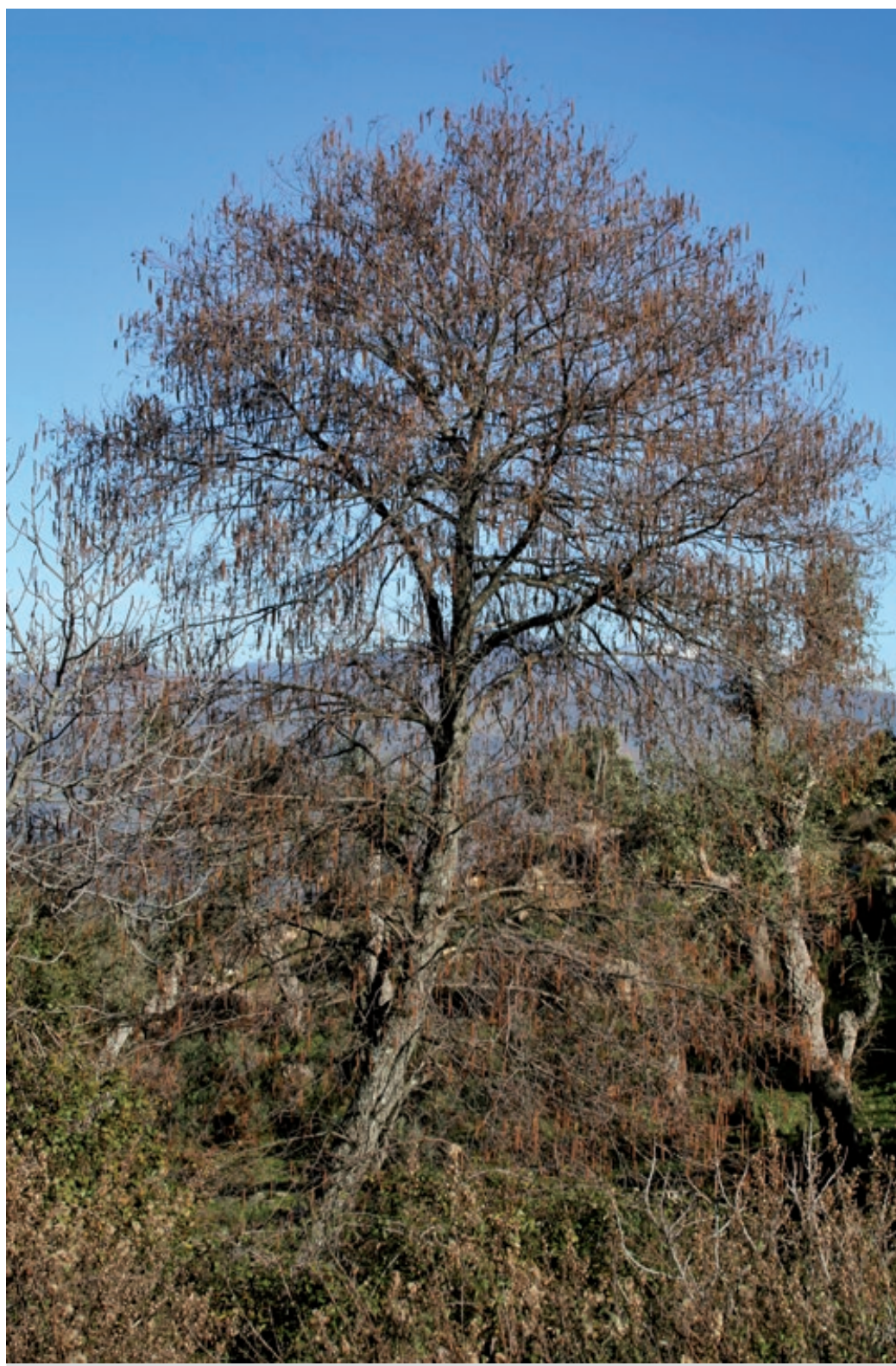

Photo 2.

Jeune Alnus glutinosa dans un petit cours d'eau à Tayenza (Rif occidental) en hiver pendant la floraison.

Young Alnus glutinosa in a small stream in Tayenza (Western Riff in winter during flowering.

Photo M. Ater. 


\section{Méthodes}

\section{Cartographie des peuplements}

La cartographie des populations à A. glutinosa est basée sur la démarche habituelle de la cartographie forestière (Rondeux, 1999) avec des adaptations relatives aux peuplements résiduels et des ripisylves (Bensettiti, 1995). L'exploration de l'aire naturelle de l'aulne glutineux a été planifiée en se basant sur les cartes d'aménagement forestier et les données bibliographiques disponibles. Étant donné que l'espèce est considérée comme rare (Fennane et Ibn Tattou, 1998 ; Hammada et al., 2002 ; Fennane, 2017), nous avons procédé à une enquête auprès des agents forestiers et de la population locale (particulièrement les bergers) pour optimiser nos campagnes de terrain. Les résultats des enquêtes ont permis d'orienter les prospections vers les cours d'eau où l'espèce a été déjà observée. Une combinaison de la carte des ressources en eau superficielle (ABHL, 2011) et de la lithologie a été réalisée à l'aide du logiciel ArcMap (ArcGis 10.3), pour identifier les cours d'eau permanents sur substrats siliceux, conditions considérées comme optimales pour l'aulne. Ensuite, la cartographie a été affinée par arpentage terrestre et complétée par analyse des photos aériennes de la mission photographique de 2003 et des images satellites sur l'application Google Earth Pro.

\section{Choix des sites représentatifs}

Compte tenu de la nature fragmentée des aulnaies marocaines, il est opportun de délimiter des unités géographiques représentatives des peuplements pour réaliser l'étude des caractéristiques dendrométriques. Ainsi, un site représentatif correspondrait à une station située en forêt avec un peuplement à aulne glutineux présentant une densité minimale de $3 \mathrm{~m}^{2}$ de ripisylves par hectare et située au sein d'une unité hydrographique (sous bassin versant) avec une géologie relativement homogène.

Les unités hydrographiques ont été délimitées automatiquement avec l'outil Watershed du logiciel ArcMap (ArcGis 10.3) pour atteindre le seuil de densité fixé précédemment. Les noms attribués aux unités correspondent aux noms reconnus localement.

\section{Typologie des peuplements}

Une typologie physionomique simplifiée a été adoptée pour caractériser la structure des peuplements. Elle est basée sur trois critères : la composition, le recouvrement de la strate arborée et le régime du peuplement. Pour la composition, deux classes ont été retenues : peuplement pur à aulne (Ag), et peuplement mixte avec Prunus lusitanica L. (Ag-Pl). Un peuplement arborescent est considéré pur lorsque l'essence ciblée a un recouvrement supérieur ou égal à $75 \%$. Pour l'estimation des recouvrements, sachant que ce type de formation se présente en bandes linéaires étroites, nous avons utilisé les recouvrements linéaires (Rondeux, 1999 ; Delassus et al., 2014). Les classes de recouvrement retenues sont les suivantes : dense, notée (1) pour un recouvrement de plus de $50 \%$; densité moyenne, notée (2) pour un recouvrement entre $50 \%$ et $25 \%$; claire, notée (3) pour un recouvrement entre $25 \%$ et $10 \%$; éparse, notée (4) pour un recouvrement linéaire compris entre $10 \%$ et $5 \%$. Enfin, pour le régime forestier, deux types ont été considérés : le régime de la futaie désigné par (f) et celui du taillis désigné par (t).

\section{Caractéristiques dendrométriques des peuplements}

\section{Échantillonnage}

Pour chaque site, un inventaire systématique a été réalisé pour 20 à 28 placettes correspondant chacune à un segment de $30 \mathrm{~m}$ de longueur avec répartition égale entre les deux rives. Le nombre de placettes a été déterminé à partir d'un inventaire préliminaire de dix placettes suivant la méthode de Lanly (1963) basée sur l'utilisation du coefficient de variation et de l'erreur type du paramètre mesuré. Pour chaque placette, nous avons relevé les données relatives à la densité et aux paramètres dendrométriques des arbres. La hauteur a été mesurée à l'aide d'un dendromètre Blume Leiss-BL6 et le diamètre des troncs avec celle d'un compas forestier positionné à une hauteur de 1,30 m.

\section{Structure des peuplements}

La structure des peuplements à $A$. glutinosa a été analysée sur la base de la distribution des diamètres des arbres. La distribution de Weibull est couramment adoptée dans ce type d'approche (Geary et al., 1985 ; Rondeux, 1999 ; Kebenzikato et al., 2014) car elle offre une grande souplesse en matière de modélisation. À cet effet, elle a été utilisée pour représenter la structure théorique de chaque peuplement sur la base de la fonction de densité de probabilité (Rondeux, 1999) :

$f(x)=\frac{c}{b}\left(\frac{x-a}{b}\right)^{c-1} e^{-\left(\frac{x-a}{b}\right)}$ (équation 1)

où $x$ est le diamètre des arbres, a est le paramètre d'origine, $b$ est le paramètre d'échelle lié à la valeur centrale des diamètres, c est le paramètre de forme lié à la structure en diamètres. Le paramètre d'origine (a) a été fixé à $7 \mathrm{~cm}$, envisagé comme valeur seuil au stade perchis où les individus peuvent être considérés comme des arbres (Rondeux, 1999). L'estimation des paramètres b et c a été effectuée avec le logiciel de statistique SPSS, version 20 par l'ajustement à une fonction non linéaire et l'évaluation de la corrélation par le coefficient de Spearman.

\section{Traitement des données}

Pour les différents paramètres mesurés, nous avons calculé les moyennes, écarts-types et coefficients de variation. Les comparaisons intersites ont été testées par l'analyse de la variance et le test de Duncan pour les comparaisons multiples des moyennes. Les analyses statistiques des données, y compris les corrélations et l'ajustement à la distribution de Weibull, ont été réalisées par les logiciels SPSS version 20 et $\mathrm{R}$ studio version 3.4.0. 


\section{Résultats et discussion}

\section{Aire de répartition de l'aulne glutineux au Maroc}

Pour délimiter l'aire de l'aulne glutineux, nous nous sommes basés sur une approximation de l'aire potentielle (figure 1) définie a priori en se basant sur les données disponibles de l'écologie, la chorologie et les affinités biogéographiques de l'espèce.

$\mathrm{Au}$ Maroc, les populations d'aulnes sont localisées dans la chaîne rifaine, plus particulièrement dans ses parties occidentale et centrale (Benamar, 2005). Du point de vue écologique, ce taxon privilégie les bioclimats subhumides à perhumides à hiver chaud à frais au sens de Emberger (1955). Il s'agit donc d'altitudes moyennes à élevées correspondant aux niveaux supérieurs du thermoméditerranéen et inférieurs du mésoméditerranéen (Benabid, 1982). Le fait qu'il s'agisse d'une espèce riparienne, préférant les substrats acides, permet de mieux cerner son habitat et limiter son aire potentielle aux cours d'eau d'altitude moyenne à substrat schisto-gréseux du Rif centro-occidental. Bien que les données sur l'écologie et la répartition de cette espèce soient relativement rares, la chorologie supposée de l'espèce se limite à deux zones floristiques, le Tangérois et le Rif centro-occidental (Fennane et Ibn Tattou, 2005), qui correspondent bien à l'aire potentielle que nous avons définie. La nature de l'habitat est également confirmée car l'espèce a été observée uniquement dans les ripisylves (Ennabili et Ater, 1996 ; Ater et al., 2008). Benamar (2005) a localisé des sites naturels de l'aulne dans les affluents de l'Oued Laou au niveau de la chaîne gréseuse de la région de Chefchaouen (figure 1). Les données des arpentages de localisation in situ ont été traitées pour produire une carte de localisation des peuplements à $A$. glutinosa (figure 2). Cette carte représente les sites où les peuplements à aulnes ont été identifiés et géoréférencés. Elle correspondrait à l'aire de répartition actuelle de l'espèce. Il apparaît que l'aulne glutineux est présent exclusivement dans un habitat rivulaire alors qu'en Algérie il peut occuper également, comme c'est le cas en Europe, des habitats relevant de marais (Belouahem-Abed et al., 2012 ; Toubal et al., 2014).

Les peuplements recensés s'étalent sur un gradient altitudinal où ils peuvent atteindre des altitudes relativement élevées, jusqu'à 1581 m au niveau du Jbel Khézana et 1603 m à Tayenza. D'une manière générale, les peuplements remarquables se situent dans une tranche altitudinale comprise entre 500 et 1100 m d'altitude. Cependant, nous avons observé des peuplements de basse altitude, comme c'est le cas à l'Oued Lakhmis, entre 78 et 240 m. Mais les aulnaies y sont très fragmentées et éparses avec des arbres très distants les uns des autres. Certains pieds isolés ont été observés pratiquement au niveau de la mer, comme c'est le cas dans la partie basse de la plaine alluviale de l'Oued Laou. Sur le plan physionomique, les peuplements d'aulnes se présentent sous formes d'alignements fragmentés colonisant les rives des cours d'eau. Dans le Rif occidental, ils occupent essentiellement des substrats de grés numidiens, alors que vers l'est, dans le Rif central, des peuplements

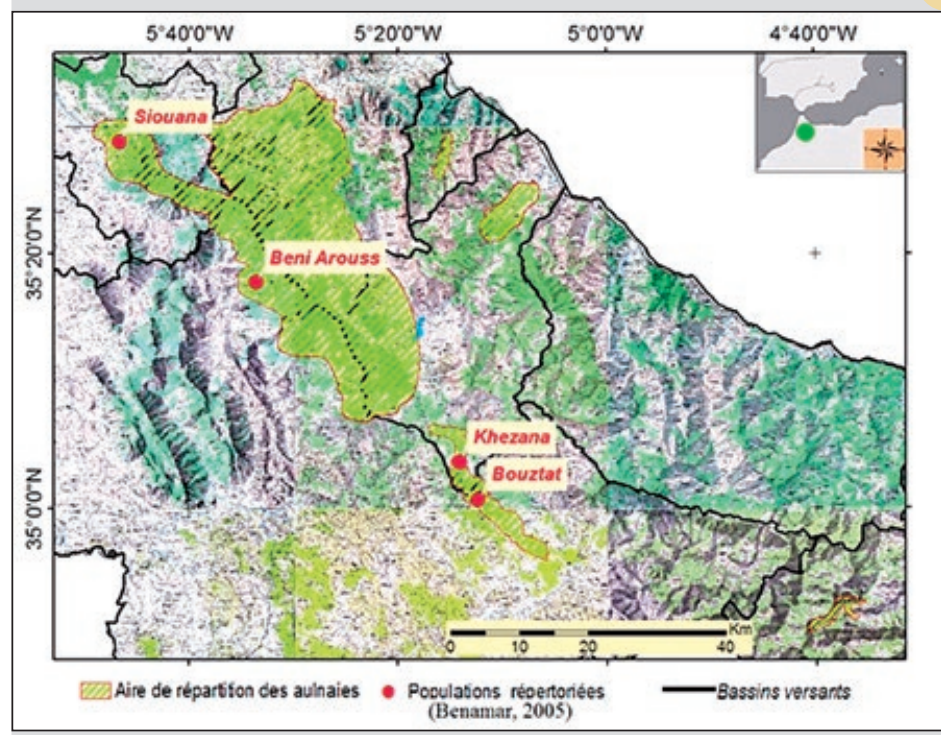

Figure 1.

Aire de répartition potentielle de l'aulne glutineux, Alnus glutinosa (L.) Gaertn., sur fond topographique correspondant au montage des feuilles topographiques de Tanger, Tétouan, Larache, El Ksar Lakbir, Chefchaouen, Jebba, Beni Boufrah, Ouazzane, Zoumi et Ghafsay au 1/100 000.

Potential distribution area of the black alder, Alnus glutinosa (L.) Gaertn., on a topographic background corresponding to the topographic sheets of Tangier, Tetouan, Larache, El Ksar Lakbir, Chefchaouen, Jebba, Beni Boufrah, Ouazzane, Zoumi and Ghafsay at the scale of 1:100 000.

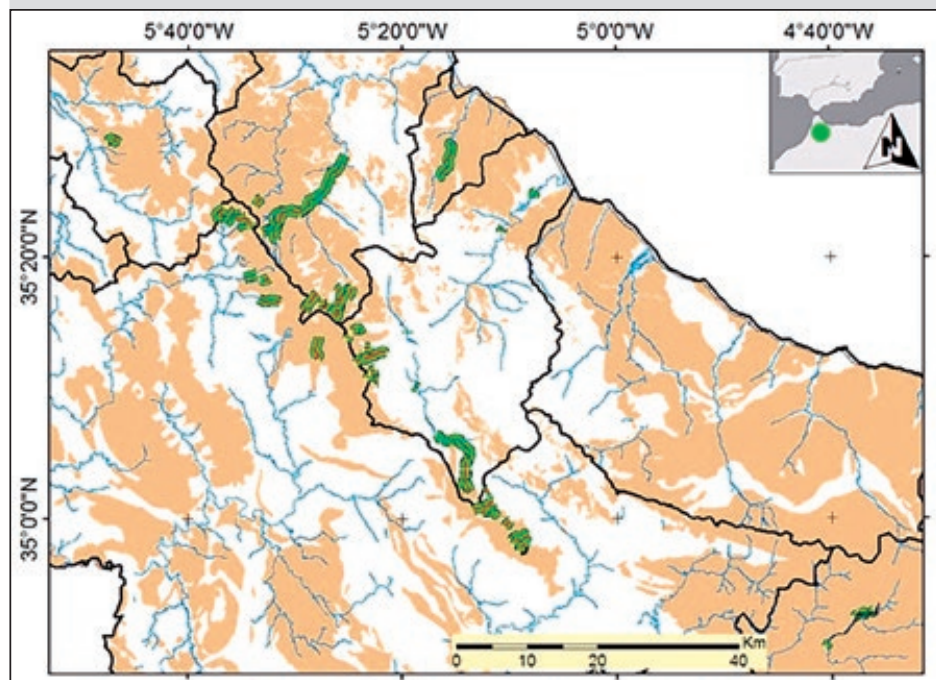

Réseau hydrographique $\longrightarrow$ thus gintinosa - Bassins versants

Figure 2.

Cartographie des peuplements inventoriés d'aulnes glutineux, Alnus glutinosa (L.) Gaertn.

Mapping of inventoried stands of black alder, Alnus glutinosa (L.) Gaertn.

moins étendus sont installés sur d'autres types de substrats comme les schistes. D'une manière générale, ces cours d'eau sont très pentus, étroits et à forts débits pendant la saison humide. La plupart sont des affluents de cours d'eau plus importants situés à des altitudes plus basses. Étant 


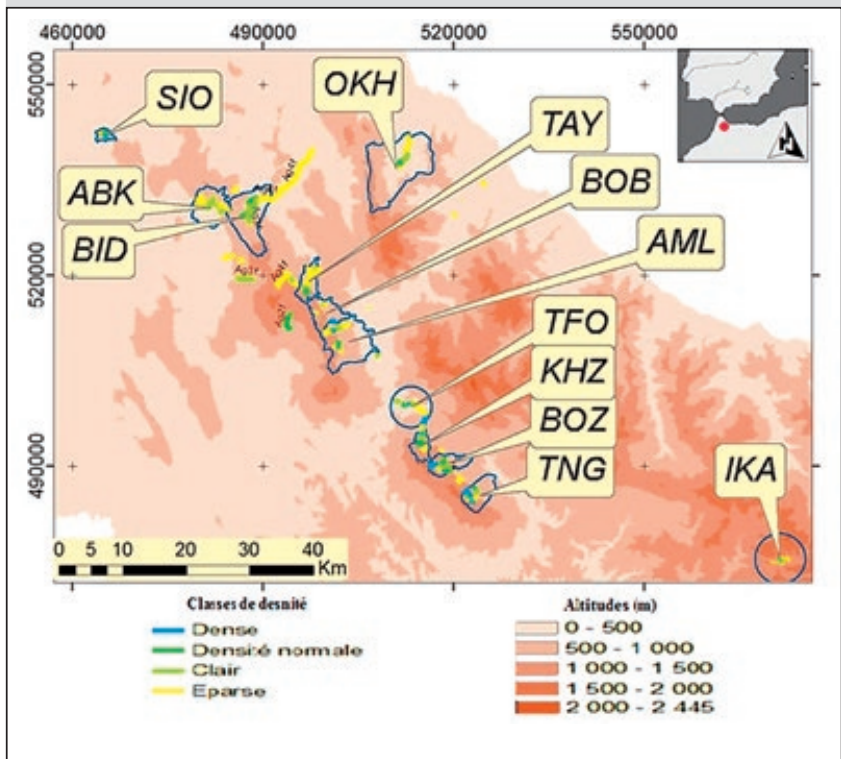

Figure 3.

Carte de localisation de sites remarquables et typologie des peuplements à Alnus glutinosa (L.) Gaertn. Pour le code des peuplements, voir le tableau I.

Location map of outstanding sites and stand typology of Alnus glutinosa (L.) Gaertn. For stand codes see Table I. donné ces caractéristiques hydrologiques, les lits des cours d'eau sont généralement étroits avec des berges pentues. Ainsi, les ripisylves sont peu larges, la plupart ne dépassant pas $3 \mathrm{~m}$ de part et d'autre, mis à part certaines parties du réseau où le lit peut s'élargir, comme c'est le cas pour l'Oued Laou (Ater et al., 2008). Du point de vue de l'étagement altitudinal des formations végétales, les formations à aulnes sont remplacées en altitude vers l'amont par des formations à Prunus lusitanica et vers le bas par des formations à Salix purpurea ou Nerium oleander à l'aval.

\section{Reconnaissance et délimitation de sites représentatifs}

L'aspect fragmenté et dispersé des aulnaies rend difficile le choix des sites pour la caractérisation des peuplements. Il fallait repérer et délimiter des tronçons de ripisylves avec des densités appréciables en aulnes glutineux. En d'autres termes, il s'agissait de délimiter des sites remarquables de référence pour la caractérisation des peuplements. Pour cela, nous avons tenu compte de la répartition géographique des unités hydrographiques et de l'accessibilité des sites. Ainsi, nous avons identifié douze sites que nous estimons représentatifs des peuplements d'aulnes dans la région (figure 3 ). Ces sites ont été nommés en se basant sur la toponymie et les noms utilisés localement. Pour chaque site, les principales données hydrographiques, climatiques et relatives aux sols ont été rassemblées (tableau I, annexe 1). Ces sites sont caractérisés par

Tableau I.

Caractéristiques physiques et bioclimatiques des sites de référence reconnus dans l'aire d'étude.

Physical and bioclimatic characteristics of recognised reference sites in the study area.

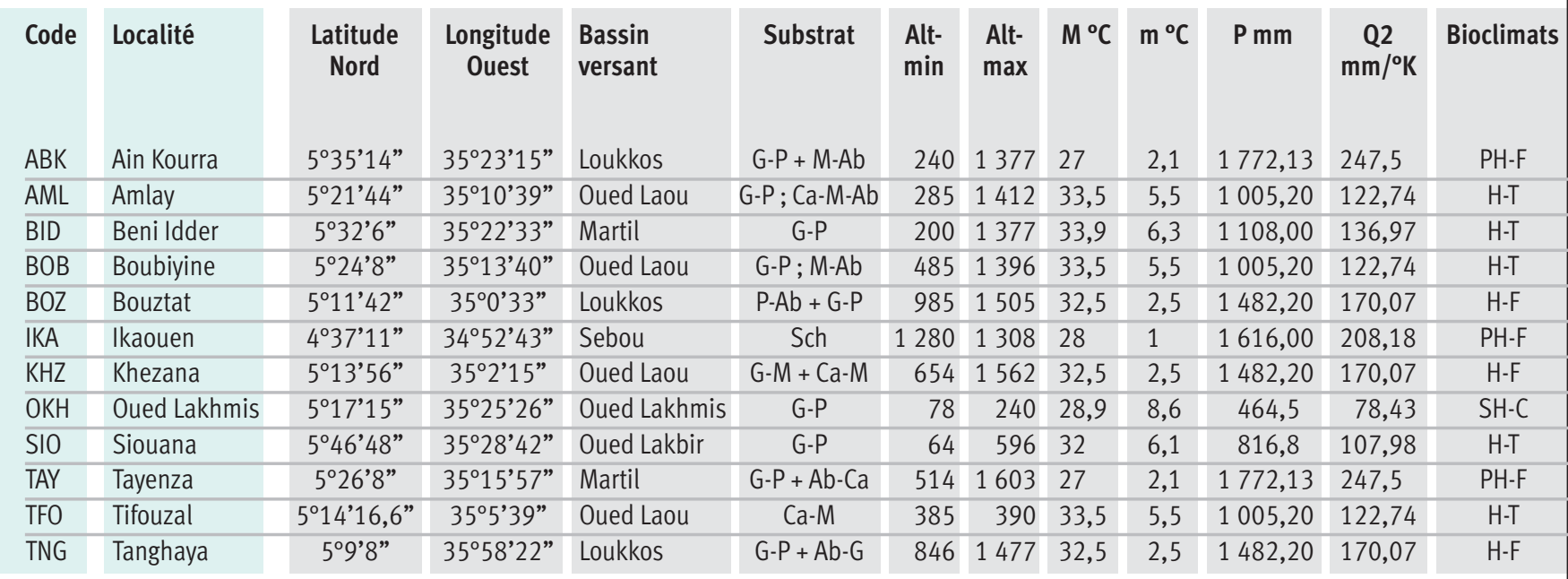

Substrat. G : grès ; P : pélites ; $A b$ : argile bariolée, $C a$ : calcaire ; $M$ : marne ; Sch : schiste. Climat. $M{ }^{\circ} \mathrm{C}$ : moyenne des maximas du mois le plus chaud de l'année; $\mathrm{m}^{\circ} \mathrm{C}:$ moyenne des minimas du mois le plus froid de l'année; $\mathrm{P}:$ moyenne des précipitations annuelles en mm ; Q2 : quotient pluviométrique d'Emberger. Source : Agence des bassins hydrauliques de Loukkos (ABHL).

Alt-min : altitude à la base de la population; Alt-max : altitude au sommet de la population. Bioclimat. PH : perhumide ; $\mathrm{H}$ : humide ; $\mathrm{SH}$ : subhumide ; variants : hiver : $\mathrm{F}=$ frais ; $\mathrm{T}=$ tempéré $; \mathrm{C}=$ chaud. 
Tableau II.

Typologie et caractérisation des peuplements de référence à Alnus glutinosa.

Typology and characterisation of reference stands of Alnus glutinosa.

\begin{tabular}{|c|c|c|c|c|c|c|c|c|c|c|c|}
\hline \multirow[t]{2}{*}{ Peuplement } & \multicolumn{4}{|c|}{$\begin{array}{c}\text { Futaie pure } \\
\text { (longueur }(\mathrm{m}) / \text { nombre de tronçons) }\end{array}$} & \multirow[b]{2}{*}{ Ag1t } & \multicolumn{2}{|c|}{ Taillis pur } & \multirow{2}{*}{$\begin{array}{l}\text { Peuplement } \\
\text { mixe } \\
\text { Ag3f-Pl3f }\end{array}$} & \multirow[t]{2}{*}{ Total } & \multirow{2}{*}{$\begin{array}{l}\text { Longueur } \\
\text { moyenne } \\
\quad(\mathrm{m})\end{array}$} & \multirow[t]{2}{*}{$\begin{array}{c}\text { Densité } \\
\text { (pieds/100 m) }\end{array}$} \\
\hline & Ag1f & $\mathrm{Ag} 2 \mathrm{f}$ & $\mathrm{Ag} 3 \mathrm{f}$ & $\mathrm{Ag} 4 \mathrm{f}$ & & Ag2t & Ag4t & & & & \\
\hline ABK & & 97 / 1 & $4148 / 8$ & $5108 / 8$ & & & & & $9353 / 17$ & 550,18 & $6,43 \pm 4,54$ \\
\hline$\overline{A M L}$ & $185 / 1$ & $1277 / 3$ & $2200 / 2$ & $3342 / 8$ & & $60 / 1$ & & & 7064 / 15 & 470,93 & $11,67 \pm 5,33$ \\
\hline$\overline{B I D}$ & & 4022 / 2 & $6560 / 4$ & $4166 / 2$ & & & & & $14748 / 8$ & 1843,50 & $9,7 \pm 6,91$ \\
\hline $\mathrm{BOZ}$ & $1695 / 1$ & $1324 / 2$ & $3007 / 2$ & $1461 / 3$ & $101 / 1$ & & & & $7588 / 9$ & 843,11 & $18,19 \pm 8,47$ \\
\hline IKA & & $263 / 1$ & $689 / 2$ & $601 / 2$ & & & & & $1553 / 5$ & 310,6 & $10,83 \pm 7,67$ \\
\hline $\mathrm{KHZ}$ & $1603 / 4$ & $1089 / 3$ & $1322 / 4$ & $1452 / 2$ & & & & & $5466 / 13$ & 420,46 & $17,33 \pm 6,53$ \\
\hline$\overline{\mathrm{OKH}}$ & & $925 / 1$ & $1945 / 2$ & $4164 / 3$ & & & & & 7034 / 6 & 1172,33 & $11,33 \pm 8,33$ \\
\hline SIO & 682 / 2 & $329 / 2$ & $406 / 1$ & 727 / 1 & & & & & 2144 / 6 & 357,33 & $13,33 \pm 4,67$ \\
\hline$\%$ & 12,86 & 15,81 & 32,25 & 36,81 & 0,13 & 0,52 & 0,81 & 0,82 & 100,00 & & \\
\hline
\end{tabular}

$\mathrm{Ag}$ et Ag-Pl désignent la composition pure et mixte des peuplements. Les chiffres de 1 à 4 correspondent aux classes de recouvrement. Les lettres $\mathrm{f}$ et $\mathrm{t}$ désignent le régime en futaie ou taillis. Pour plus de détails, voir le chapitre Méthodes.

des bioclimats perhumides ou humides, mis à part celui de l'Oued Lakhmis qui a un bioclimat subhumide (annexe 1). En effet, les précipitations sont généralement abondantes, variant de $1000 \mathrm{~mm}$ à $1770 \mathrm{~mm}$, à l'exception du site de l'Oued Lakhmis où elles sont nettement inférieures avec seulement $464 \mathrm{~mm}$ (tableau I). D’une manière générale, les hivers sont de type frais à tempéré et les températures estivales restent modérées. La lithologie est dominée par des substrats acides (gréseux ou schisteux).

\section{Typologie des peuplements}

L'approche adoptée pour la typologie des peuplements d'aulnes dans les sites de référence a permis d'en préciser les principales caractéristiques comme la nature du régime forestier, la composition, la taille et les densités (figure 3, tableau II). En ce qui concerne le régime forestier, comme il s'agit de peuplements naturels issus de semis, ils sont constitués quasiment de peuplements en futaie (97,7\%). Cependant, on peut observer des peuplements en taillis dans des sites perturbés où des coupes ont été pratiquées ; mais ils sont rares et représentent moins de $2 \%$ des peuplements. Ils ont été observés dans trois sites, principalement à Tayenza et en moindre importance à Bouztat et Amlay. Ceci ne veut pas dire, toutefois, que les autres sites sont non impactés par les coupes.

D’une manière générale, la composition des peuplements est à peu près pure, l'aulne représentant l'espèce dominante de la strate arborescente. Cependant, il existe des peuplements mixtes avec Prunus lusitanica. Mais ceux-ci sont relativement rares et représentent moins de $1 \%$ des peuplements. Ces peuplements mixtes se situent au niveau de la limite altitudinale supérieure d'A. glutinosa et forment la transition avec des ripisylves pures à $P$. lusitanica en altitude.

Les peuplements d'aulnes se présentent sous forme de formations en couloirs étroits et discontinus avec une structure fragmentée. Dans les sites de référence, les aulnaies sont constituées de fragments d'une longueur moyenne de $670 \mathrm{~m}$ dont le nombre varie entre 5 à lkaouen et 17 à Ain Kourra. Cependant, il faut prendre en considération l'importance de la variabilité de ces paramètres aussi bien à l'intérieur des sites qu'entre les sites (tableau II). Ainsi, la longueur moyenne des fragments par site varie entre $310 \mathrm{~m}$ à lkaouen et 1843 m à Beni Idder. De même, la taille globale des peuplements par site varie beaucoup entre les sites, cela dans un large intervalle compris entre une longueur totale de $1553 \mathrm{~m}$ pour lkaouen et $14748 \mathrm{~m}$ pour Beni Idder.

Les peuplements épars (classe Ag4) avec 37,6\% et les ripisylves claires (classe Ag3) avec 33,1\% sont de loin les plus représentatifs en totalisant $70,7 \%$ des peuplements (tableau II, figure 3). Ces deux catégories s'apparentent à des peuplements très fragmentés avec des arbres distants à isolés. Les peuplements à densité normale (classe Ag2) ou dense (Ag1), qui s'apparentent à des structures en couloir continu ou discontinu, représentent seulement $29 \%$ des peuplements. D’une manière générale, la majorité des peuplements denses se trouvent en moyenne altitude sur des expositions Nord-Est. 


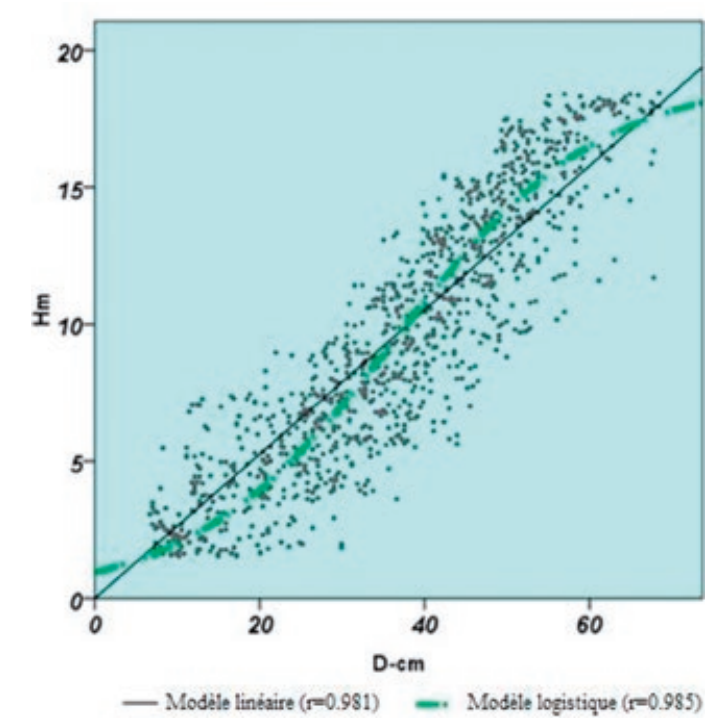

Figure 4.

Corrélations entre hauteur $(\mathrm{Hm})$ et diamètre (D-cm) des arbres suivant les deux modèles linéaire et logistique. $r$ est le coefficient de corrélation.

Correlations between tree height $(\mathrm{Hm})$ and diameter $(D-\mathrm{cm})$ according to both linear and logistic models. $r$ is the correlation coefficient.

Pour la structure des peuplements et en complément aux données relatives aux recouvrements, les résultats de l'inventaire ont permis d'estimer les densités (tableau II). La densité moyenne varie entre une valeur minimale de $6,19 \pm 4,29$ pieds $/ 100 \mathrm{~m}$ pour Ain Kourra et une valeur maximale de 28,33 $\pm 8,33$ pieds $/ 100 \mathrm{~m}$ pour Tanghaya.

\section{Caractéristiques dendrométriques des peuplements}

D’une manière générale, les paramètres dendrométriques reflètent la croissance des arbres. Cette dernière peut être considérée comme la résultante du potentiel physiologique déterminé génétiquement et la réponse aux conditions de l'environnement correspondant à la plasticité phénotypique. La hauteur des arbres est un paramètre important en foresterie car celui-ci renseigne d'une manière directe sur la qualité et l'état d'un peuplement. Le diamètre des arbres est un paramètre plus facile à mesurer, qui renseigne non seulement sur la croissance mais aussi, indirectement, sur l'âge des arbres. Il est parfois difficile d'estimer la hauteur des arbres, d'où l'intérêt des corrélations entre hauteur et diamètre, qui ont fait l'objet de nombreuses études pour différentes essences (Castedo Dorado et al., 2005 ; Newton et Amponsah, 2007). Pour les peuplements étudiés, la corrélation entre ces deux paramètres est hautement significative avec des coefficients de corrélation supérieurs à 0,9 pour les deux modèles, linéaire et logistique (figure 4). Avec un coefficient de détermination $r^{2}=0,81$, la régression permet une bonne estimation de la hauteur des arbres à partir de leur diamètre.

La hauteur moyenne des arbres dans le nord du Maroc est de 9,53 4,62 m (tableau III, figure 5). C'est une hauteur sensiblement inférieure à celles observées pour d'autres peuplements dans l'ouest de la Méditerranée. En effet, dans la péninsule ibérique, la hauteur moyenne observée en Espagne est de 14,45 × 5,04 m (Rodríguez-González, 2004) et 14,05 \pm 4,33 m au Portugal (Rodríguez-González et al., 2014). De même, en Algérie où se trouve la deuxième zone refuge d'aulnes en Afrique du Nord, la hauteur moyenne est

Tableau III.

Caractéristiques dendrométriques des peuplements de référence.

Dendrometric characteristics of reference stands.

\begin{tabular}{|c|c|c|c|c|c|c|}
\hline \multirow[t]{2}{*}{ Peuplement } & \multicolumn{2}{|c|}{ Nombre } & \multirow{2}{*}{$\begin{array}{l}\text { Diamètre } \\
\text { Moyen }(\mathrm{cm})\end{array}$} & \multirow[b]{2}{*}{ CV (\%) } & \multirow{2}{*}{$\begin{array}{l}\text { Hauteur } \\
\text { Moyenne }(\mathrm{m})\end{array}$} & \multirow[b]{2}{*}{ CV (\%) } \\
\hline & Placettes & Arbres & & & & \\
\hline ABK & 28 & 54 & $35,17 \pm 13,9 b$ & 39,51 & $6,59 \pm 2,89 b$ & 43,83 \\
\hline$\overline{A M L}$ & 20 & 70 & $37,38 \pm 12,68 b$ & 33,93 & $7,09 \pm 3,84 b$ & 54,1 \\
\hline BID & 22 & 64 & $28,76 \pm 15,25 a$ & 53 & $6,98 \pm 3,25 b$ & 46,51 \\
\hline BOB & 20 & 101 & $38,79 \pm 12,63 b$ & 32,56 & $10,3 \pm 4,73 c$ & 45,94 \\
\hline $\mathrm{BOZ}$ & 24 & 131 & $39,84 \pm 12 b$ & 30,11 & $10,91 \pm 4,29 c$ & 39,27 \\
\hline IKA & 20 & 65 & $41,37 \pm 13,43 b$ & 32,48 & $10,33 \pm 4,94 \mathrm{c}$ & 47,85 \\
\hline KHZ & 20 & 104 & $28,68 \pm 15,76 a$ & 54,94 & $8,87 \pm 4,64 c$ & 52,3 \\
\hline $\mathrm{OKH}$ & 20 & 68 & $25,05 \pm 15,44 a$ & 61,63 & $6,35 \pm 3,43 b$ & 54,12 \\
\hline $\mathrm{SIO}$ & 20 & 80 & $38,29 \pm 14,24 b$ & 37,18 & $10,36 \pm 4,41 c$ & 42,56 \\
\hline TAY & 26 & 75 & $38,55 \pm 14,27 b$ & 37,01 & $9,41 \pm 4,8 \mathrm{c}$ & 51 \\
\hline TFO & 20 & 87 & $39,59 \pm 13,05 b$ & 32,95 & $9,03 \pm 4,29 c$ & 47,53 \\
\hline TNG & 20 & 85 & $41,47 \pm 12,32 b$ & 29,72 & $12,26 \pm 4,17 a$ & 34,02 \\
\hline Moyenne totale & & & $36,77 \pm 14,67$ & 39,89 & $9,53 \pm 4,62$ & 48,48 \\
\hline ANOVA & & & $F=7,045 ;$ Sig $: 0,000$ & & $F=6,630 ; \operatorname{Sig}=0,000$ & \\
\hline
\end{tabular}

Moyen \pm écart-type. Les lettres différentes ( $a, b, c)$ signifient que les moyennes sont significativement différentes selon le test de comparaisons multiples des moyennes de Duncan. CV : coefficient de variation. ANOVA : analyse de la variance à 1 critère. $\mathrm{F}$ : rapport de variance. Sig : significatif à $\mathrm{P}<0,001$. 

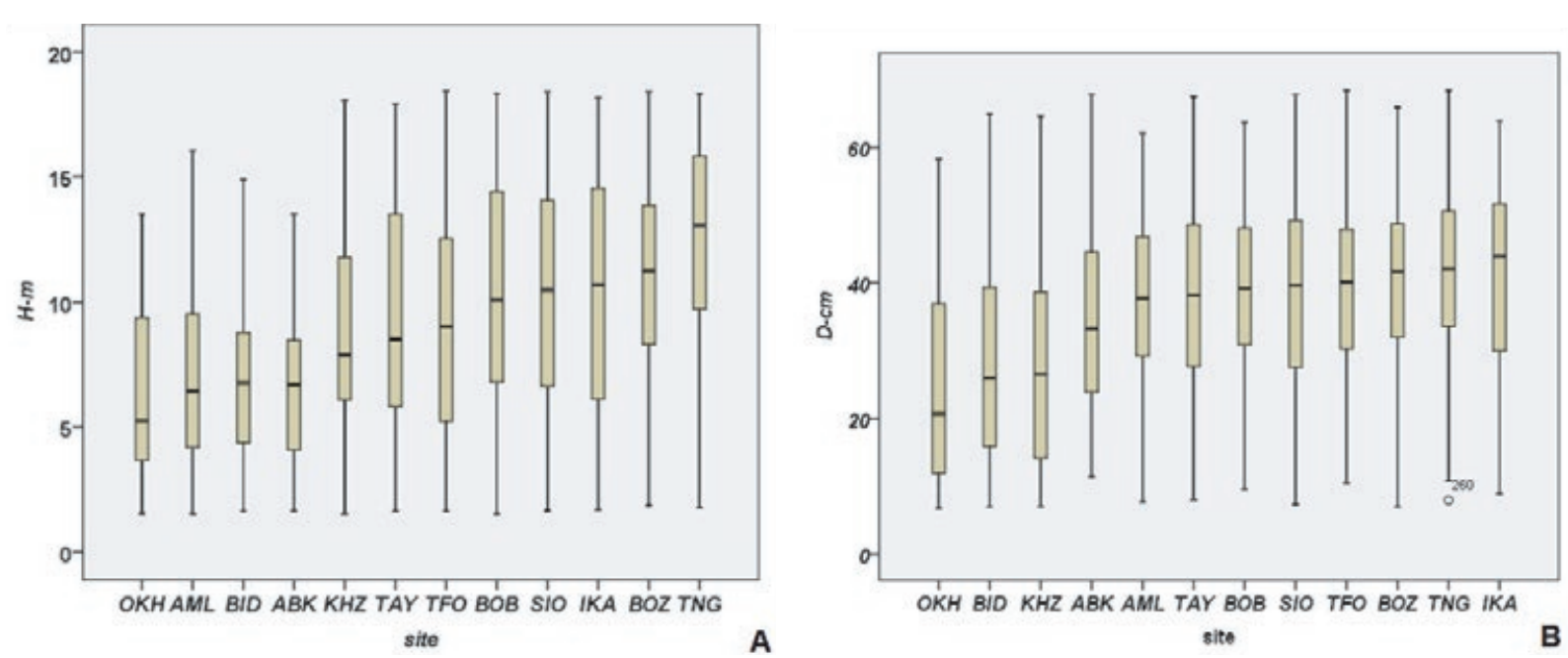

Figure 5.

Comparaison des distributions des hauteurs (A) et diamètres (B) des arbres d'Alnus glutinosa (L.) Gaertn.

Pour le code des peuplements, voir le tableau I.

Comparison of height (A) and diameter (B) distributions of Alnus glutinosa (L.) Gaertn. For stand code see Table I.

de 12,82 \pm 4,50 m (Belouahem-Abed, 2012). La hauteur inférieure des aulnaies marocaines s'expliquerait par le type d'écosystème et l'impact des activités humaines. L'habitat de l'aulne au Maroc correspond à des ripisylves présentes la plupart du temps dans des milieux fortement anthropisés. Cependant, la moyenne générale des hauteurs cache une grande variabilité aussi bien entre peuplements qu'à l'intérieur de ceux-ci. En effet, les moyennes estimées par peuplement varient entre $6,35 \pm 3,43 \mathrm{~m}$ comme valeur minimale à l'Oued Lakhmis et 12,26 \pm 4,17 m comme valeur maximale à Tanghaya (tableau III, figure 5). L'analyse de la variance montre que les variations intersites sont hautement significatives (tableau III). Ces variations correspondent à un gradient de taille opposant deux pôles : d'un côté des peuplements bas avec des arbres dont la hauteur est inférieure ou égale à $7 \mathrm{~m}$ et, de l'autre, des peuplements hauts dont la hauteur des arbres est de $12 \mathrm{~m}$ avec un seul site, Tanghaya. Les autres peuplements forment un groupe intermédiaire où les hauteurs sont comprises entre $8,87 \mathrm{~m}$ pour le peuplement de Khézana et 10,91 m pour le peuplement de Bouztat. Cette classification en trois groupes est validée par le test de comparaisons multiples des moyennes (tableau III). Les variations intrapeuplement, estimées par les coefficients de variation, se sont montrées plus importantes dans les peuplements à hauteurs basses comme l'Oued Lakhmis avec $54,12 \%$ de variation et, inversement, moins importantes pour les peuplements à hauteurs élevées comme Tanghaya, avec seulement 34,02 \%. Ainsi, les peuplements à hauteurs basses semblent moins homogènes et correspondraient à des peuplements dynamiques plus jeunes, à l'opposé des peuplements à hauteurs supérieures qui seraient plus âgés avec une régénération faible. Cette différenciation des peuplements par les hauteurs des arbres serait en relation avec la structure des peuplements, les conditions écologiques des sites et la pression anthropique occasionnée par les prélèvements de bois et feuillages pour des usages domestiques. En effet, les peuplements de hauteurs supérieures sont des peuplements denses et à recouvrements élevés avec des tronçons courts, l'inverse s'observant pour les peuplements de hauteurs basses. Du point de vue des conditions écologiques et de la qualité de l'habitat, les peuplements de hauteurs supérieures se trouvent dans des sites d'altitude avec des précipitations élevées et des conditions hydrologiques plus favorables.

Le diamètre moyen mis en évidence dans les peuplements du nord du Maroc est de l'ordre de 36,8 $\pm 14,7 \mathrm{~cm}$ (tableau III, figure 5). Il est similaire à celui observé en Espagne avec 35,2 \pm 12,4 cm (Rodríguez-González et al., 2004) et au Portugal avec 36,3 \pm 13,9 cm (Rodríguez-González et al., 2014). En Algérie, les aulnaies présentent au contraire des diamètres inférieurs, avec un diamètre moyen de 29,8 $\pm 13,9 \mathrm{~cm}$ (Belouahem-Abel, 2012). Les résultats obtenus montrent une structuration similaire à celle des données relatives à la hauteur des arbres, avec une variabilité intrasite et intersites relativement élevée. Ainsi, les diamètres moyens des arbres s'étendent le long d'un gradient entre $25,1 \mathrm{~cm}$ à l'Oued Lakhmis et 41,5 cm à Tanghaya (tableau III, figure 5). L'analyse de la variance montre que ces variations entre les peuplements sont hautement significatives, permettant une discrimination des peuplements par les diamètres des arbres. En effet, la comparaison multiple des moyennes permet de distinguer deux groupes : un groupe avec de faibles diamètres comprenant trois sites, l’Oued Lakhmis, Khézana et Beni Idder ; un groupe comprenant le reste des sites avec des diamètres supérieurs compris entre $35,2 \mathrm{~cm}$ pour Ain Kourra et $41,5 \mathrm{~cm}$ pour Tanghaya. La variation intrapeuplement est nettement plus élevée chez les peuplements de faibles diamètres et atteint $61,63 \%$ pour l'Oued Lakhmis alors qu'elle est seulement de l'ordre de 29,72\% pour le peuplement de Tanghaya à diamètres élevés. 
Les peuplements d'aulnes étudiés ont montré une forte variabilité des paramètres dendrométriques, cela malgré une aire de répartition réduite. Il se manifeste une gradation des peuplements en fonction de la taille et la grosseur des arbres. Elle correspond à une différenciation de la structuration des peuplements. Suivant les sites, les peuplements présentent des caractéristiques différentes. En altitude, sous des conditions favorables, les peuplements sont denses, plus homogènes, avec des arbres plus hauts et des diamètres élevés.

Au contraire, vers le bas, les peuplements sont épars, les arbres restent très distants, avec des arbres moins hauts et des diamètres nettement inférieurs.

\section{Structure et dynamique des populations}

Le diamètre des arbres est considéré comme un paramètre intégrateur pour décrire les propriétés structurales et démographiques d'un peuplement forestier. En effet, la distribution des diamètres des arbres traduit indirectement la distribution des âges et permet d'apprécier la structure et la dynamique des peuplements. Selon les modèles démographiques de l'écologie des populations (MacArthur et Wilson, 1967 ; Pianka, 1970), la distribution des âges permet de reconnaître différents états chez les populations de plantes en fonction de la succession et de la stratégie reproductive.

L'interprétation des distributions des diamètres permet de renseigner la dynamique des peuplements, qui peut correspondre à différents états : stable, en déclin ou dégradé (Molinas et al., 1991 ; Rondeux, 1999 ; Ajbilou et al., 2003). La structure des peuplements illustrée par la distribution des classes de diamètre (annexe 2) montre une forte variabilité et des situations bien contrastées. Dans ce sens, l'ajustement de la distribution des diamètres à la distribution théorique de Weibull (Bullock et Burkhart, 2005) permet de mieux caractériser la structure des peuplements forestiers (figure 6). Les paramètres de la distribution permettent d'interpréter les courbes et d'apprécier la dynamique des peuplements, spécialement le paramètre c qui caractérise la forme de la distribution (Hush et Scovel, 2003). Le résultat des ajustements des distributions des différents peuplements montre alors des formes variables et contrastées, que nous pouvons classer en quatre types de structures en fonction de la valeur du paramètre $c$ :

- distributions avec $c<1$ et une courbe en J inversée pour les peuplements de l'Oued Lakhmis et Khézana ; on peut parler d'une structure jardinée correspondant à un peuplement dynamique en régénération ;

- distributions avec $1<c<3,6$ et une courbe à asymétrie positive pour les peuplements Siouana, Beni Idder, Ain Kourra et Boubiyine ; ce type de distribution correspondrait à des peuplements avec un faible potentiel de régénération et une dominance d'individus de faible diamètre ;

- distributions avec $c=3,6$ et une courbe en forme de cloche pour les peuplements Tayenza, Tifouzal et Amlay; ce type de distribution caractérise des peuplements à faible potentiel de régénération ;

- distributions avec $c>3,6$ et une courbe à asymétrie négative pour les peuplements Tanghaya, Bouztat et Ikaouen; ce type de distribution caractérise généralement des peuplements dégradés à faible régénération.

S'agissant de la structuration des peuplements, on observe, d'une part, des peuplements dynamiques en régénération avec des arbres à petits diamètres fréquents dans les peuplements épars à faible densité et, d'autre part, des peuplements présentant un faible taux de régénération et la dominance d'individus à diamètres élevés au sein de peuplements généralement plus denses. Dans le premier cas, il s'agit de stades primaires de succession correspondant à des situations très différentes. Par exemple, dans le cas de l'Oued Lakhmis, il s'agit d'un site ouvert avec une ripisylve sans strate arborée et une forte dynamique hydromorphologique où les conditions écologiques ne sont pas optimales pour l'espèce. À Khézana, les conditions sont plus favorables, mais le site est fortement perturbé par des pressions anthropogènes, principalement l'intensité des coupes. Dans le second cas, la dominance des individus âgés s'expliquerait par des raisons naturelles et des facteurs exogènes. En effet, à Tanghaya et Bouztat, il s'agit de populations denses à recouvrement élevé où le recrutement d'individus juvéniles est faible. À lkaouen, la faible régénération s'expliquerait en revanche par l'état dégradé du peuplement et la forte pression anthropogène.

\section{Implications pour la conservation}

Les données réunies par cette étude, qui concernent la localisation et la taille des populations ainsi que leur structure et leur dynamique, dressent un diagnostic inquiétant sur l'état de conservation d'A. glutinosa au Maroc. En effet, la taille de la zone d'occupation réelle est extrêmement réduite par rapport à la taille d'occurrence de l'espèce (figures 1 et 2). Cette zone est incluse géographiquement au sein d'une seule division floristique, ce qui confirme le statut de taxon rare au Maroc (Fennane et Ibn Tattou, 1998). En dépit de la nature linéaire des peuplements qui rend difficile l'évaluation de la superficie occupée par l'espèce, cette dernière pourrait être estimée à un maximum de 500 km² en se basant sur une longueur totale d'environ $80 \mathrm{~km}$ (tableau II) et une largeur moyenne de $6 \mathrm{~m}$ des ripisylves. L'aire de répartition représentée par les sites de référence adoptés dans ce travail est très fragmentée, répartie sur plusieurs cours d'eau secondaires et morcelés en 116 tronçons de longueurs et structures variables (tableau II). D'un autre côté, les données dendrométriques montrent que la régénération est faible pour la majorité des peuplements (figure 6), que ce soit pour des causes naturelles ou anthropogènes. En effet, la qualité de l'habitat de cette espèce est fortement dégradée à cause des déficits hydriques aggravés par la surexploitation des ressources en eau selon une pratique courante de captage des eaux en amont par les populations riveraines. Cependant, les aulnaies du nord du Maroc occupent des paysages ruraux fortement occupés par les populations humaines. Certains sites comme Siouana, Khezana, Amlay, Boubiyine ou lkaouen hébergent des habitations rurales dispersées dans de petits hameaux, ce qui accroît les impacts de l'écimage, des parcours ou de l'agriculture. Ce type d'écosystème ressemble parfois à la 

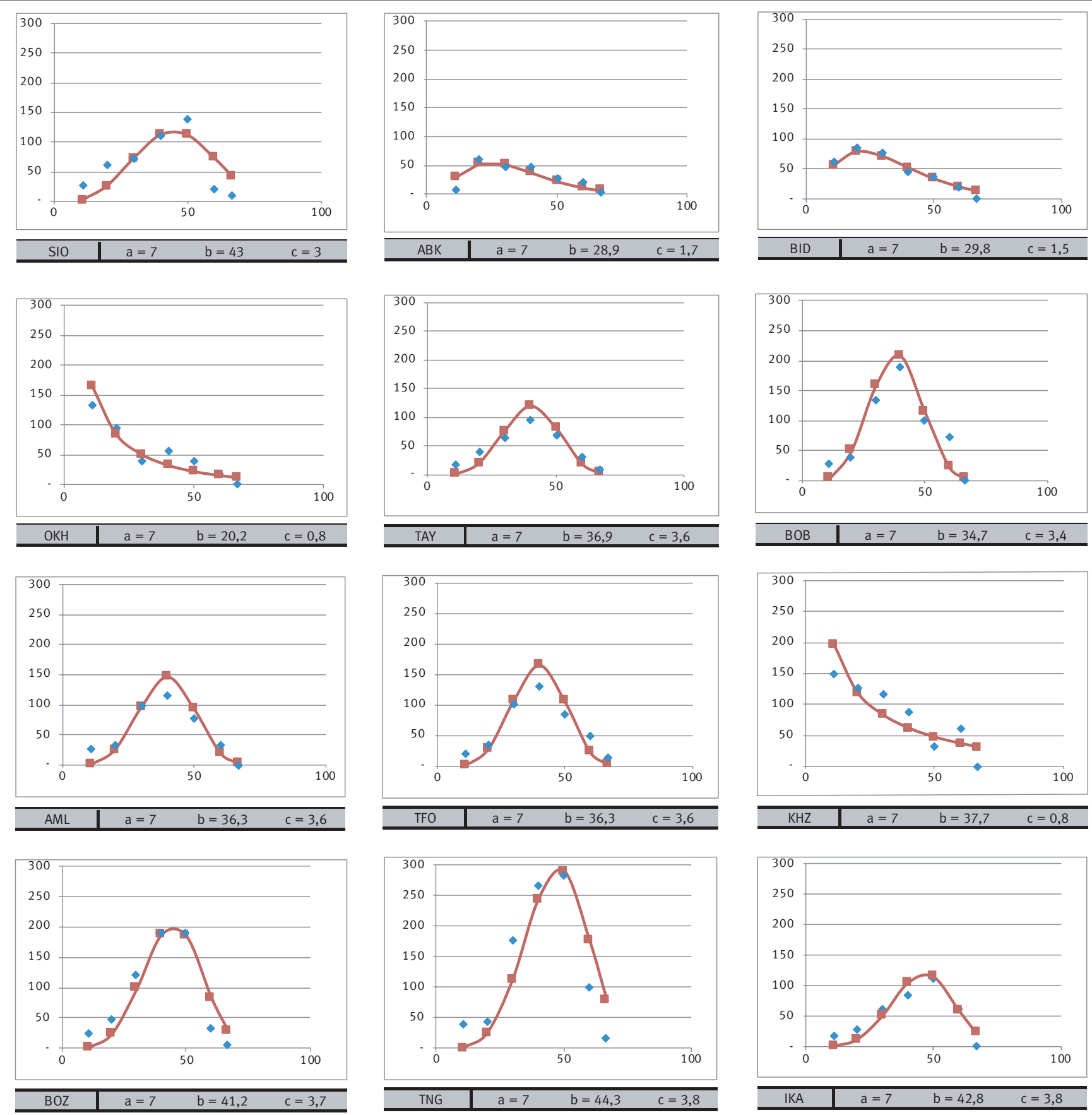

\section{Figure 6.}

Ajustement à la distribution de Weibull des fréquences (pieds/ha) en fonction des diamètres $(\mathrm{cm})$ des arbres. $a$ : paramètre d'origine ; $b$ : paramètre d'échelle ; $c$ : paramètre de forme (voir méthodes). Pour le code des peuplements, voir le tableau I. Fit to Weibull distribution of frequencies (feet/ha) versus tree diameters (cm). a: original parameter; b: scale parameter; c: shape parameter (see methods). For stand code see Table I.

forêt dite rurale ou paysanne (Simenel, 2011). Bien qu'il n'y ait pas de liste rouge officielle selon les critères de l'UICN au Maroc, Fennane a publié une telle liste. A. glutinosa y est classé dans la catégorie NT, c'est-à-dire taxon quasi menacé (Fennane, 2017). Eu égard aux données obtenues, l'état de conservation pour ce taxon reste sous-estimé. La réduction de son aire et sa fragmentation, associées à une faible régénération et une dégradation de l'habitat, plaident en faveur de son classement dans les catégories menacées des listes rouges de l'UICN (2012) en tant que taxon en danger [EN]. Le contexte des changements globaux dans la région méditerranéenne aggrave cette menace (Pachauri 
et al., 2014 ; Lionello et Scarascia, 2018), d'autant que ce taxon est fortement sensible au changement climatique (Rodríguez-González et al., 2014). La conservation de cette espèce riparienne est conditionnée par la préservation de son habitat et de sa qualité. Or les formations ripariennes au Maroc ne bénéficient d'aucun statut particulier de protection. Du point de vue légal, ces formations font partie du domaine hydraulique public géré par les agences des bassins hydrauliques, comme le stipule la loi relative à l'eau (Loi n 36-15 du 10 août 2016). Cependant, ces formations restent indirectement soumises au régime forestier bien qu'elles soient localisées dans le domaine hydraulique. L'administration des Eaux et forêts, en tant que responsable de la défense et la restauration du sol (Dahir n 1-69-170 du 25 juillet 1969), participe à l'établissement des plans d'aménagement et de gestion des bassins hydrauliques. Cet écosystème est donc à la croisée des compétences de deux administrations, sans pour autant bénéficier d'un statut ou de mesures de protection spécifiques. L'adaptation du cadre légal avec la reconnaissance d'un statut spécifique est incontournable pour assurer la protection des habitats occupés par les formations ripariennes en général et celles de $A$. glutinosa en particulier.

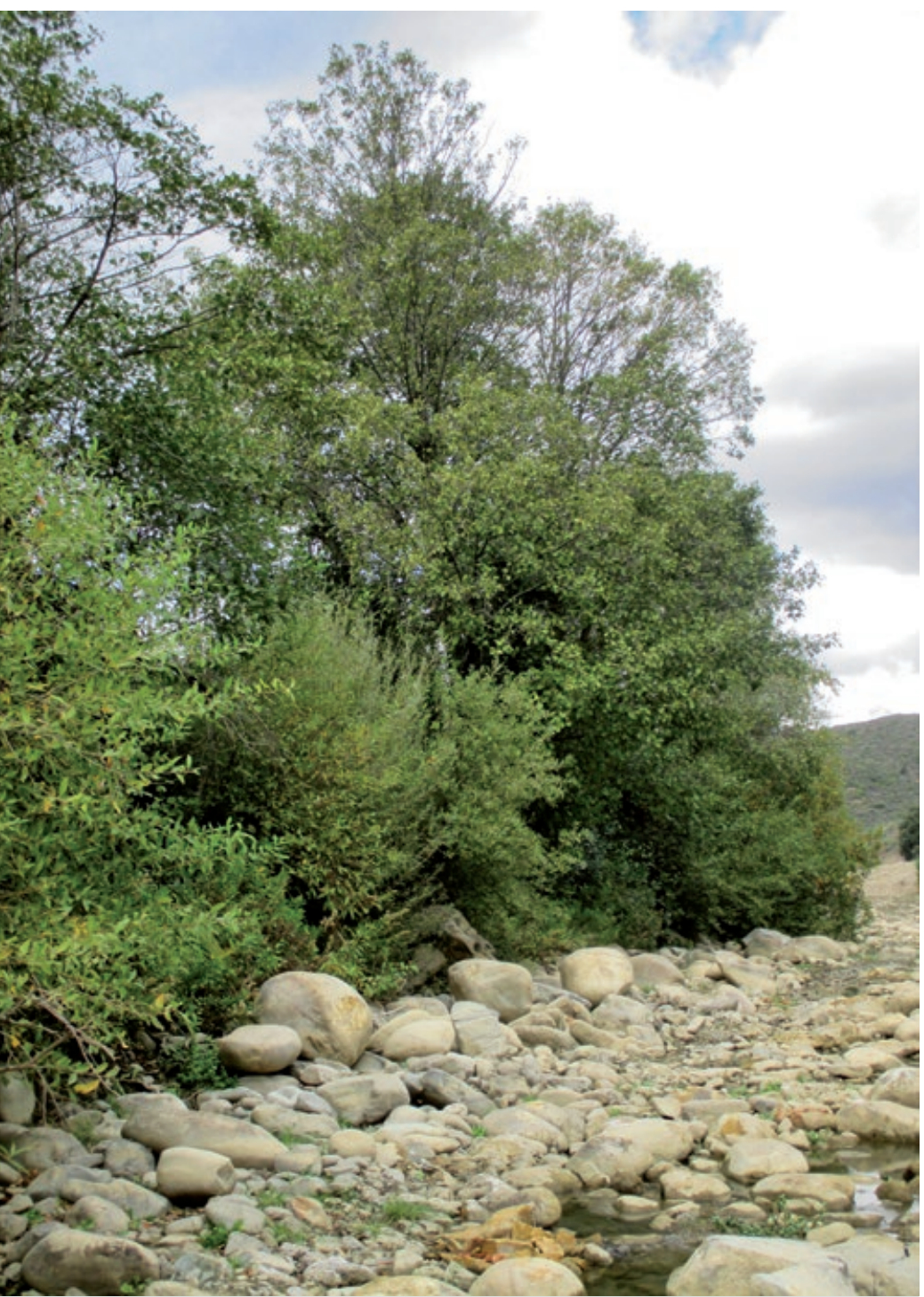

Photo 3.

Ripisylve à aulne et saules dans un affluent de l'Oued Laou (Oued Moulay Bouchta, Dar Aakobaa, Chefchaoeun). Alder and willows riparian zone in a tributary of the Oued Laou (Oued Moulay Bouchta, Dar Aakobaa, Chefchaoeun). Photo M. Ater.

\section{Annexe 1.}

Climagramme d'Emberger avec localisation des sites de référence et des stations météorologiques représentatives de la région.

Emberger climagram with location of reference sites and representative weather stations in the region.

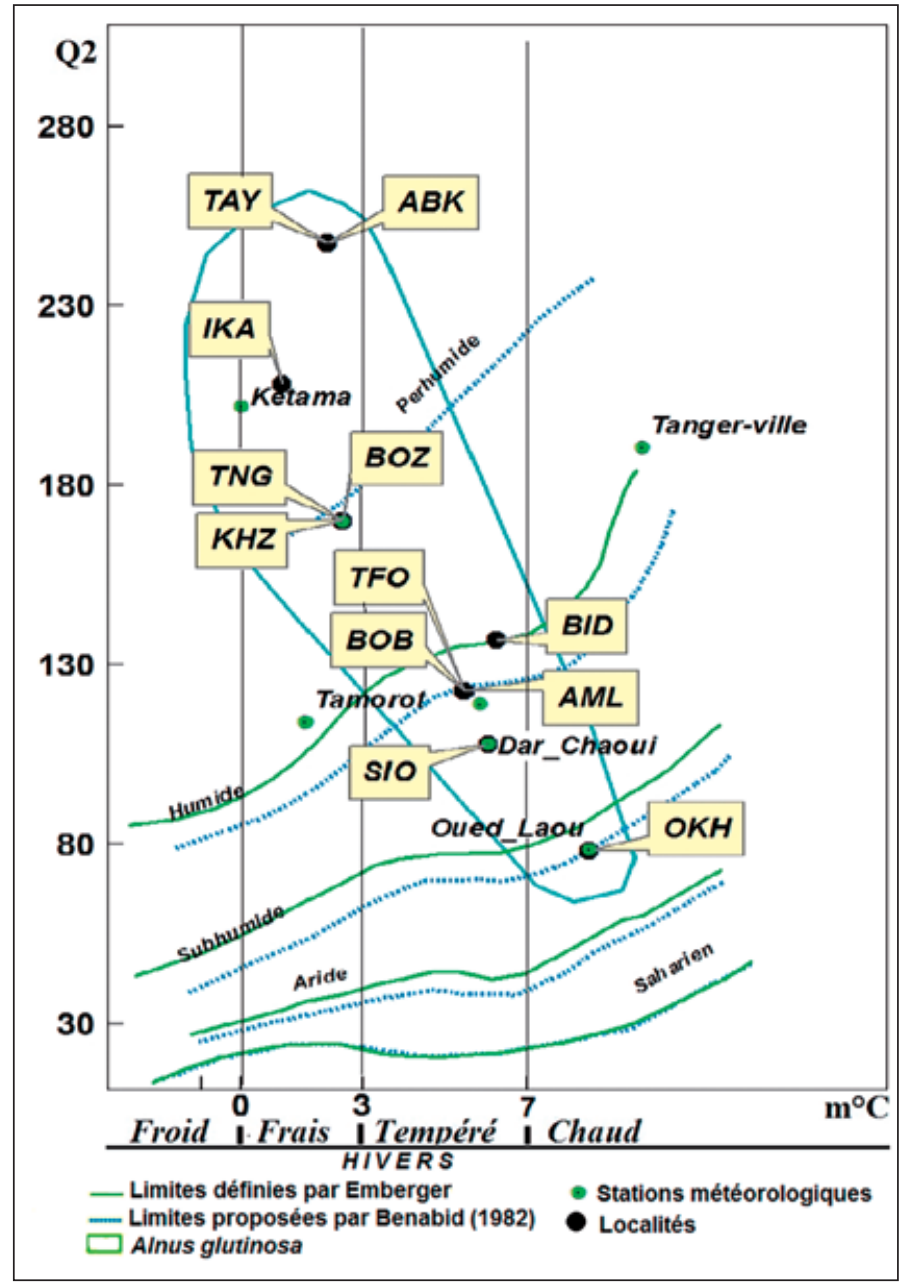


Annexe 2.

Structures de peuplements illustrées par la distribution des fréquences (pieds/ha) en fonction des classes de diamètre $(\mathrm{cm})$. Stand structures illustrated by frequency distribution (feet/ha) according to diameter classes (cm).
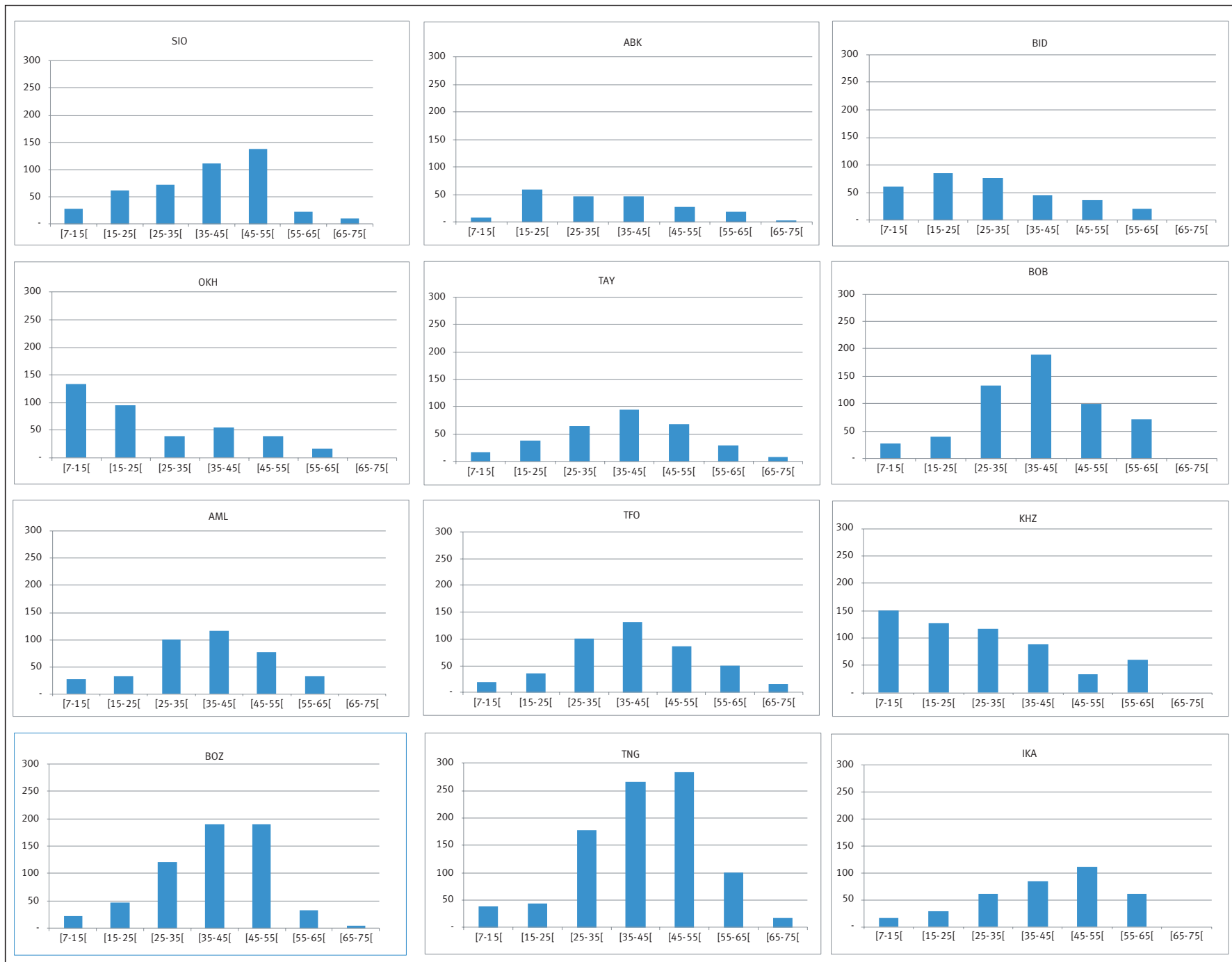

\section{Conclusion}

Les résultats obtenus constituent un apport de données quantitatives géoréférencées permettant d'avoir une bonne connaissance de la répartition et la dynamique des peuplements d'Alnus glutinosa au Maroc. Bien qu'il ne s'agisse pas d'un inventaire exhaustif des peuplements d'aulnes, il s'agit de la première délimitation et caractérisation des peuplements marocains réalisées à une échelle aussi fine pour cette espèce. En plus de la cartographie des peuplements, cette étude a permis d'en caractériser la taille, le recouvrement, la densité et divers paramètres dendrométriques.

Les peuplements d'aulnes se présentent en couloirs étroits, discontinus et fragmentés. II s'agit de peuplements en futaie où l'aulne représente l'espèce dominante de la strate arborée. Plus de $70 \%$ des peuplements sont peu denses avec des arbres distants à isolés. Les caractéristiques dendrométriques montrent des variations hautement significatives aussi bien pour le diamètre que pour la hauteur, cela aux niveaux intrasite et intersites. Ces variations sont en relation avec la densité des peuplements, les conditions écologiques et l'intensité des perturbations de l'habitat. Le type de structuration observé correspond à des dynamiques contrastées en fonction de la succession, des conditions écologiques et des perturbations. Un gradient apparaît selon quatre types de structure, gradient aux pôles duquel se présentent respectivement des peuplements peu denses, dynamiques, en régénération avec de petits diamètres, et des peuplements denses avec un faible taux de régénération et de grands diamètres.

Les données obtenues mettent en évidence un diagnostic alarmant sur l'état des peuplements et la qualité de leur habitat. Ce taxon doit être considérée comme menacé au Maroc et devrait bénéficier à court terme d'un statut de protection et d'un programme de conservation. Cela se justifierait par sa valeur patrimoniale comme taxon relictuel rare et son intérêt comme ressource génétique au regard de la singularité et de la diversité génétique des populations marocaines. 


\section{Remerciements}

Les auteurs remercient les responsables des Directions régionales des eaux et forêts et de la lutte contre la désertification du Rif et du Nord-Est au Maroc pour les moyens humains et matériels mis à leur disposition afin de faciliter les investigations et enquêtes sur le terrain.

\section{Références bibliographiques}

ABHL, 2011. Étude d'actualisation du plan directeur d'aménagement intégré des ressources en eau des bassins Loukkos, Tangérois et côtiers méditerranéens. Tétouan, Maroc, Agence du bassin hydraulique du Loukkos (ABHL).

Ajbilou R., Marañón T., Arroyo J., 2003. Distribución de clases diamétricas y conservación de bosques en el norte de Marruecos. Investigación agraria. Sistemas y Recursos Forestales, 12 (2) : 111-123. https://digital.csic.es/handle/10261/48574

Arènes J., 1951. À propos des connexions ibéro-marocaines et siculo-tunisiennes. Compte-rendu sommaire des séances de la Société de biogéographie, 241 : 67-72.

Ater M., Radi M., Kadiri M., Qninba A., 2008. Ripisylves et avifaune du bassin versant de Oued Laou. Travaux de l'Institut scientifique, Rabat, Série générale, $n^{\circ} 5$ : 27-35.

Benabid A., 1982. Bref aperçu sur la zonation altitudinale de la végétation climacique au Maroc. Ecologia Mediterranea, 8 (1-2) : 301-315. https://www.persee.fr/doc/ecmed 015387561982 num 811956

Belouahem-Abed D., 2012. Étude écologique des peuplements forestiers des zones humides dans les régions de Skikda, Annaba et El Tarf (Nord-Est algérien). Université Badji Mokhtar-Annaba, Algérie, 320 p.

Benamar S., 2005. Contribution à la réhabilitation au Maroc de l'espèce forestière Alnus Glutinosa par son étude écophysiologique et la caractérisation moléculaire de son microsymbiote diazotrophe Frankia. Thèse de doctorat d'État. Université Moulay Ismail, Faculté des sciences de Meknès, Maroc, $244 \mathrm{p}$.

Bensettiti F., 1995. Contribution à l'étude phytosociologique des ripisylves du Nord de l'Algérie. Essai de synthèse à l'échelle de la Méditerranée occidentale. Thèse, Université Paris-Sud, France, 180 p.

Bullock B. P., Burkhart H. E., 2005. Juvenile diameter distributions of loblolly pine characterized by the two-parameter Weibull function. New Forests, 29 (3): 233-244. https://doi. org/10.1007/s11056-005-5651-5

Castedo Dorado F., Barrioanta M., Parresol B. R., Álvarez Gonzalez J. G., 2005. A stochastic height-diameter model for maritime pine ecoregions in Galicia (northwestern Spain). Annals of Forest Science, 62: 455-465. https://doi. org/10.1051/forest:2005042

Delassus L., Magnanon S., Colasse V., Glémarex E., Guitton H., Laurent E., et al., 2014. Classification physionomique et phytosociologique des végétations de Basse-Normandie, Bretagne et Pays de la Loire. Brest, France, Conservatoire botanique national de Brest, $262 \mathrm{p}$.
Douda J., Doudová J., Drašnarová A., Kuneš P., Hadincová V., Krak K., et al., 2014. Migration patterns of subgenus Alnus in Europe since the last glacial maximum: A systematic review. PLoS ONE, 9 (2). https://doi.org/10.1371/journal. pone.0088709

Emberger L., 1955. Une classification biogéographique des climats. Recueil des Travaux des Laboratoires de Botanique, Géologie et Zoologie (Faculté des Sciences, Montpellier), série Botanique, 7 : 3-43.

Ennabili A., Ater M., 1996. Flore (Pteridophyta et Spermatophyta) des zones humides du Maroc Méditerranéen : Inventaire et écologie. Acta Botanica Malacitana, 21 : 221-239. https://doi.org/10.24310/abm.v21i0.8677

Fennane M., Ibn Tattou M., 1998. Catalogue des plantes vasculaires rares, menacées ou endémiques du Maroc. Bocconea, $8: 1-243$.

Fennane M., Ibn Tattou M., 2005. Flore vasculaire du Maroc : Inventaire et chorologie. Vol. 1. Pteridophyta, Gymnospermae, Angiospermae. Travaux de l'Institut scientifique, Rabat, série Botanique, $n^{\circ}$ 37, vol. 1, 483 p.

Fennane M., 2017. Éléments pour un Livre rouge de la flore vasculaire du Maroc. Fascicule 4. Basellaceae - Buxaceae (Version 1, septembre 2017). Tela-Botanica, 29 p. https:// www.tela-botanica.org/wp-content/uploads/2017/09/ Tela-Bot LivreR-FVM Fasc-4-sept.-2017.pdf

Geary D. N., Rennolls K., Rollinson T. J. D., 1985. Characterizing diameter distributions by the use of the Weibull distribution. Forestry, 58 (1): 57-66. https://doi.org/10.1093/ forestry/58.1.57

Hammada S. M., Dakki M., Ibn Tattou A., Ouyahya A., Fennane M., 2002. Catalogue de la flore des zones humides du Maroc. Université Mohammed V-Agdal, Institut scientifique, Bulletin de l'Institut scientifique, 24 : 1-59.

Havrdová A., Douda J., Krak K., Vít P., Hadincová V., Zákravský P., et al., 2015. Higher genetic diversity in recolonized areas than in refugia of Alnus glutinosa triggered by continent-wide lineage admixture. Molecular Ecology, 24: 47594777. https://doi.org/10.1111/mec.13348

Hush D., Scovel C., 2003. Polynomial-time decomposition algorithms for support vector machines. Machine Learning, 51 (1): 51-71. https://doi.org/10.1023/A:1021877911972 Kebenzikato A. B., Wala K., Dourma M., Atakpama W., Dimobe K., Pereki H., et al., 2014. Distribution et structure des parcs à Adansonia digitata L. (baobab) au Togo (Afrique de l'Ouest). Afrique Science, 10 (2) : 434-449. https://www. ajol.info/index.php/afsci/article/view/109682

Lanly J.-P., 1963. Détermination du taux d'échantillonnage d'un inventaire statistique en fonction de la densité des peuplements à inventorier. Annales de l'École Nationale des Eaux et Forêts et de la Station de Recherches et Expérimentations, 20 (2) : 291-304.

Lepais O., Muller S. D., Ben Saad-Limam S., Benslama M., Rhazi L., Belouahem-Abed D., et al., 2013. High genetic diversity and distinctiveness of rear-edge climate relicts maintained by ancient tetraploidisation for Alnus glutinosa. PLoS ONE, 8 (9). https://doi.org/10.1371/journal. pone.0075029 
Lionello P., Scarascia L., 2018. The relation between climate change in the Mediterranean region and global warming. Regional Environmental Change, 18: 1481-1493. https:// doi.org/10.1007/s10113-018-1290-1

Mandák B., Krak K., Vít P., Pavlíková Z., Lomonosova M. N., Habibi F., et al., 2016. How genome size variation is linked with evolution within Chenopodium sensu lato. Perspectives in Plant Ecology, Evolution and Systematics, 23: 18-32. https://doi.org/10.1016/i.ppees.2016.09.004

MacArthur R. H., Wilson E. O., 1967. The theory of island biogeography. Princeton, NJ, USA, Princeton University Press, 224 p.

McVean D. N., 1953. Biological flora of the British isles. Alnus glutinosa (L.) Gaertn. Journal of Ecology, 41 (2): $447-$ 466. https://doi.org/10.2307/2257070

Médail F., Quézel P., 1999. Biodiversity hotspots in the Mediterranean basin: Setting global conservation priorities. Conservation Biology, 13 (6): 1510-1513. https://doi. org/10.1046/i.1523-1739.1999.98467.x

Molinas L. M., Oliva M., Caritat A., 1991. Estructura y conocimiento de seis parcelas del alcornocal gerundense. Studia Oecologica, 8: 159-170. https://dialnet.unirioja.es/servlet/ articulo?codigo $=78861$

Newton P. F., Amponsah I. G., 2007. Comparative evaluation of five height-diameter models developed for black spruce and jack pine stand-types in terms of goodness-of-fit, lack-of-fit and predictive ability. Forest Ecology and Management, 247 (1): 149-166. https://doi.org/10.1016/j. foreco.2007.04.029

Pachauri R. K., Allen M. R., Barros V. R., Broome J., Cramer W., Christ R., et al., 2014. Climate Change 2014: Synthesis Report. Contribution of Working Groups I, II and III to the Fifth Assessment Report of the Intergovernmental Panel on Climate Change. Geneva, Switzerland, IPCC, 151 p. https:// hdl.handle.net/10013/epic.45156

Pianka E., 1970. On r- and K-Selection. The American Naturalist, 104: 592-597.

Quézel P., 2000. Réflexions sur l'évolution de la flore et de la végétation au Maghreb méditerranéen. Paris, France, Ibis Press, $117 \mathrm{p}$.

Rodríguez-González P. M., Campelo F., Albuquerque A., Rivaes R., Ferreira T., Pereira J. S., 2014. Sensitivity of black alder (Alnus glutinosa [L.] Gaertn.) growth to hydrological changes in wetland forests at the rear edge of the species distribution. Plant Ecology, 215 (2): 233-245. https://doi. org/10.1007/s11258-013-0292-9

Rodríguez-González P. M., Ferreira M. T., Rego P. R., 2004. Northern Ibero-Atlantic wetland woods: Vegetation types and within-stand structure. Forest Ecology and Management, 203 (1-3): 261-272. https://doi.org/10.1016/j. foreco.2004.07.068

Rondeux J., 1999. La mesure des arbres et des peuplements forestiers. Gembloux, Belgique, Les Presses agronomiques de Gembloux, 521 p.

Simenel R., 2011. Comment domestiquer une forêt sans les hommes ? Techniques \& Culture, 56 : 224-247. https://doi. org/10.4000/tc.5918
Toubal O., Boussehaba A., Toubal A., Samraoui B., 2014. Biodiversité méditerranéenne et changements globaux : cas du complexe de zones humides de Guerbès-Senhadja (Algérie). Physio-Géo, 8 : 273-295. https://doi.org/10.4000/physio-geo.4217

UICN, 2012. Catégories et Critères de la Liste rouge de l'UIC : Version 3.1. $2^{\mathrm{e}}$ édition. Gland, Suisse, Cambridge, RoyaumeUni, UICN, 32 p.

\begin{tabular}{|c|c|}
\hline \multicolumn{2}{|c|}{ Ennouni et al. - Contribution des auteurs } \\
\hline Rôle du contributeur & Noms des auteurs \\
\hline Conceptualisation & M. Ater \\
\hline Gestion des données & H. Ennouni, A. Sahli \\
\hline Analyse formelle & H. Ennouni, M. Ater \\
\hline $\begin{array}{l}\text { Acquisition } \\
\text { du financement }\end{array}$ & M. Ater \\
\hline $\begin{array}{l}\text { Enquête et } \\
\text { investigation }\end{array}$ & H. Ennouni, A. Sahli \\
\hline Méthodologie & H. Ennouni, M. Ater \\
\hline Gestion de projet & M. Ater, H. Ennouni \\
\hline Ressources & M. Ater, H. Ennouni \\
\hline Logiciels & H. Ennouni \\
\hline Supervision & M. Ater \\
\hline Validation & M. Ater, H. Ennouni \\
\hline Visualisation & H. Ennouni \\
\hline $\begin{array}{l}\text { Écriture - Préparation } \\
\text { de l'ébauche originale }\end{array}$ & M. Ater, H. Ennouni, A. Sahli \\
\hline $\begin{array}{l}\text { Écriture - Révision } \\
\text { et édition }\end{array}$ & M. Ater, H. Ennouni \\
\hline
\end{tabular}

Bois et Forêts des Tropiques - Revue scientifique du Cirad (c) Bois et Forêts des Tropiques (c) Cirad
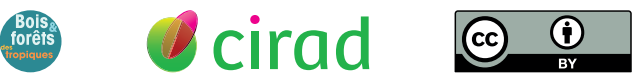

Cirad - Campus international de Baillarguet, 34398 Montpellier Cedex 5, France - Contact : bft@cirad.fr - ISSN : L-0006-579X 